\title{
UPDATES ON THE DIAGNOSIS AND TREATMENT OF PEDIATRIC HEPATOBLASTOMA WITH PULMONARY METASTASES
}

\author{
Oana Tatiana Miron'1, Anca Maria Adavidoaiei², Vlad-Adrian Afrasanie ${ }^{3}$, \\ Doina Mihaila ${ }^{4}$, Ingrith Miron ${ }^{1,5}$ \\ 1 "Gr. T. Popa" University of Medicine and Pharmacy, Iasi \\ ${ }^{2}$ Infectious Disease Department No. 2, "Sf. Parascheva” Infectious Diseases Hospital, Iasi \\ ${ }^{3}$ Medical Oncology Department, Regional Institute of Oncology, Iasi \\ ${ }^{4}$ Pathology Department, “Sf. Maria” Emergency Children's Hospital, Iasi \\ ${ }^{5}$ Hematology/Oncology Department, “Sf. Maria” Emergency Children’s Hospital, Iasi
}

\begin{abstract}
Although the frequency of hepatoblastoma is low, it is the most common primary malignant liver tumor in children. The prognosis of the disease has improved considerably in the last decades due to oncological and surgical treatment advances. Nonetheless, tumors which are diagnosed at an advanced stage still have a poor prognosis. We present the case of a 33-month-old child, diagnosed with high-risk hepatoblastoma (pulmonary metastases) in February 2014. Surgery was performed and the tumor completely removed. Afterwards, chemotherapy treatment was initiated according to a modified SIOPEL-4 protocol - the chemotherapy blocks which should have been administered before surgery were received after the operation. After finishing the chemotherapy, the thoraco-abdominal CT scan indicated a complete response to treatment. The periodic evaluation of the patient revealed the absence of local tumor recurrence, the absence of metastases, and a Lansky performance status of 80 up to now. The administration of targeted molecular therapies, liver transplant, and new chemotherapy drugs could improve the prognosis for patients with high risk hepatoblastoma in the future. Also, modifying the chemotherapy protocols could be considered an option in the achievement of this goal.
\end{abstract}

Keywords: pulmonary metastases, SIOPEL-4 protocol, complete tumor response

\section{INTRODUCTION}

Hepatoblastoma is the most common malignant tumor of the liver in children even though it has a low annual incidence of 1,5 cases per million children aged $<15$ years. (1) The disease prognosis has significantly improved for the past four decades thanks to chemotherapy and surgical techniques advances which can ensure the complete resection of the tumor. (2) Societe Internationale d'Oncologie Pediatrique - Epithelial Liver Tumor Study Group (SIOPEL) recommends the use of tumor resection together with chemotherapy pre and postoperative, depending of the stage of the disease in the moment of its diagnosis.
Despite the development of therapeutic methods, efforts are being made to find new ways of treatment which would benefit the patients with hepatoblastoma in an advanced stage. Among these are new chemotherapeutic agents (irinotecan, oxaliplatin), targeted molecular therapies, and new chemotherapy protocols. (1)

\section{CASE REPORT}

We present the case of a 33-month-old male child, patient in the Hematology/Oncology Department from “Sf. Maria” Emergency Children's Hospital, hospitalized in January 2014 for the investigation of a tumoral mass of the epigastric region.

Corresponding author:

Oana Tatiana Miron, "Gr. T. Popa" University of Medicine and Pharmacy, 16 Universitatii Street, lasi

E-mail: miron_oana85@yahoo.com 
Anamnesis and clinical exam revealed: abdominal pains, nausea, and vomiting; the palpation of the abdomen showed the presence of a tumoral mass in the epigastric region with hard consistency and a diameter of $\sim 10 \mathrm{~cm}$, and normal superjacent teguments; liver $5 \mathrm{~cm}$ below the costal margin; cough, polypnea and harsh bilateral vesicular murmur. The other organs and systems were within parameters.

Biological investigations proved: inflammatory syndrome (fibrinogen=522 mg/dl), hepatic cytolysis syndrome (GOT $155 \mathrm{U} / \mathrm{L})$, thrombocytosis (thrombocytes $=442.000 / \mathrm{mm}^{3}$ ), and alpha fetoprotein (AFP) > $30.000 \mathrm{U} / \mathrm{L}$.

Imagistic investigations described during an abdominal ultrasound a well-defined heterogeneous tumoral mass with a diameter of $\sim 7 / 8 \mathrm{~cm}$ in a retro-
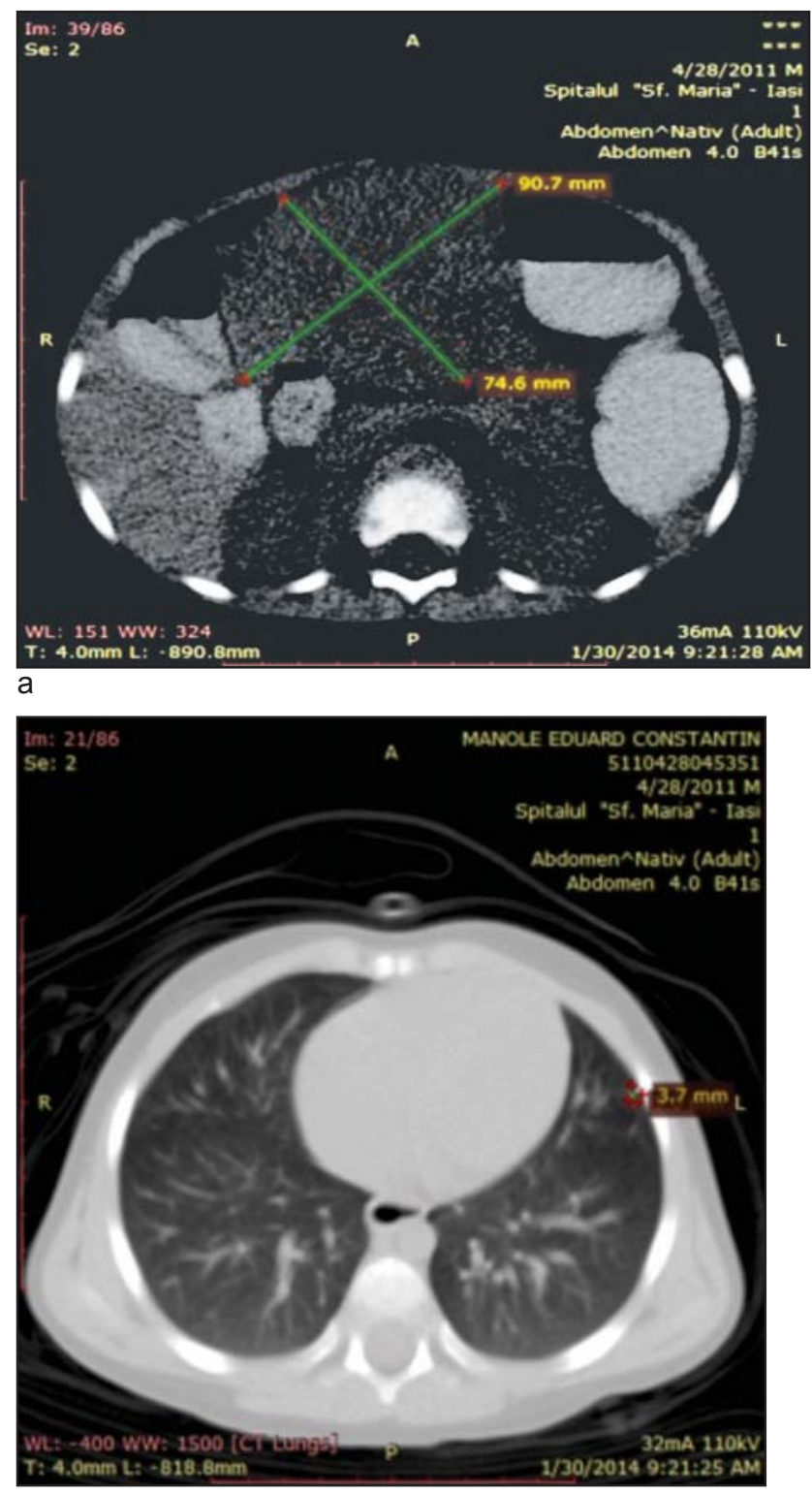

C peritoneal position with a predominantly left-sided development. The thoraco-abdomino-pelvin CT scan describes a heterogeneous expansive tumoral mass with dimensions of $7 / 9 / 9 \mathrm{~cm}$, with multiple areas of intratumoral necrosis, located on the left liver lobe (Fig. 1 - a,b), and infracentimetric radiopaque nodules located subpleural in the left hemithorax with a characteristic aspect of secondary lesions (Fig. 1 - c,d)

The caregivers were explained the therapeutic possibilities, the risks and benefits of the SIOPEL-4 protocol, and the initiation of pre-operatory chemotherapy was suggested. They refused antineoplastic therapy and chose the surgical procedure. During the surgical intervention, in February 2014, the complete excision of the tumor was performed within oncological safety limits.

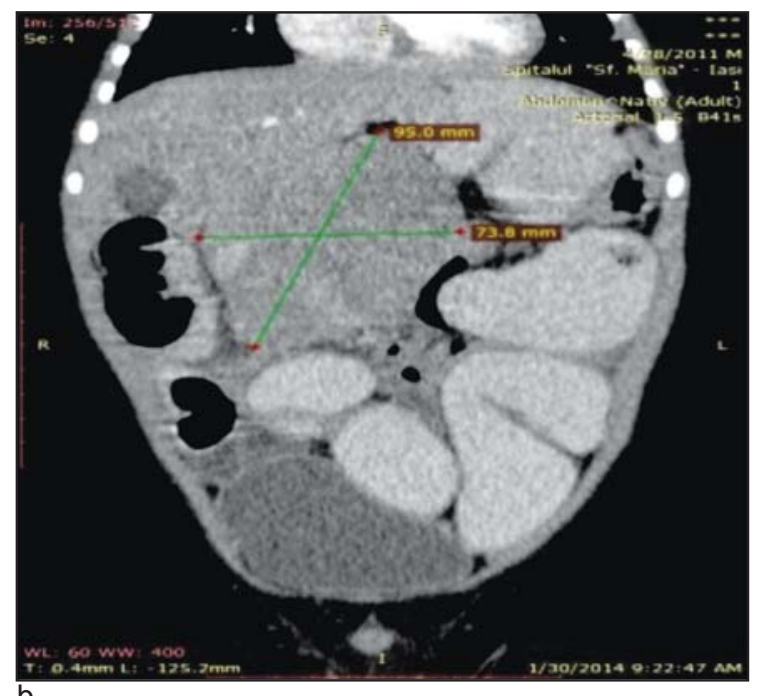

$\mathrm{b}$

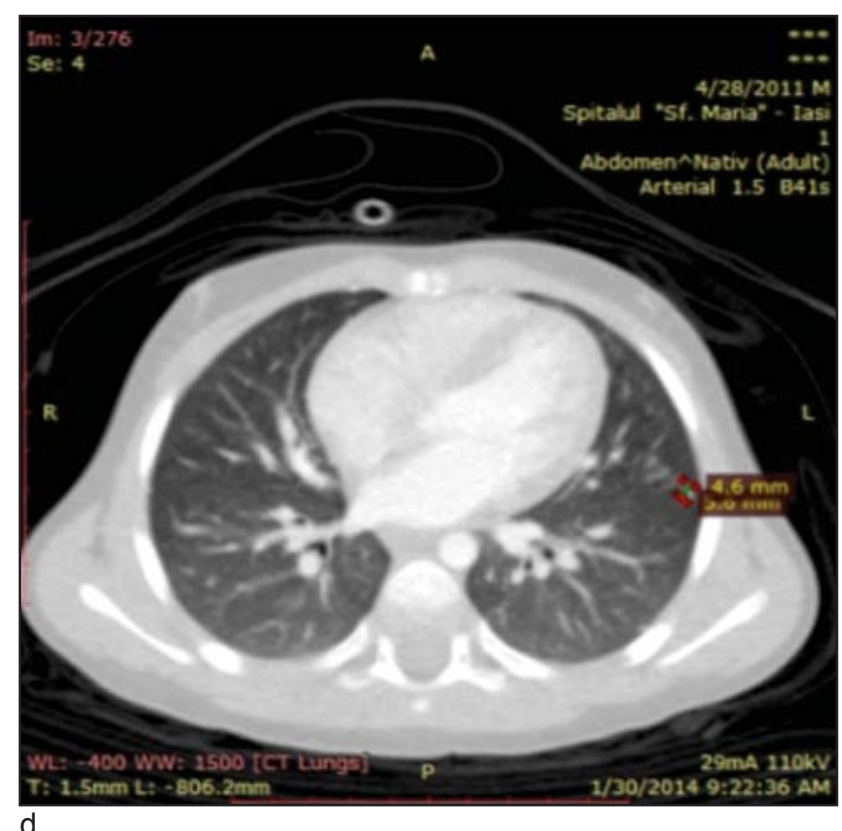

FIGURE 1 - a. Tumoral mass left liver lobe - sagittal section; b. Tumoral mass left liver lobe - cranial-caudal view; c, d. Infracentimetric pulmonary secondary lesions 


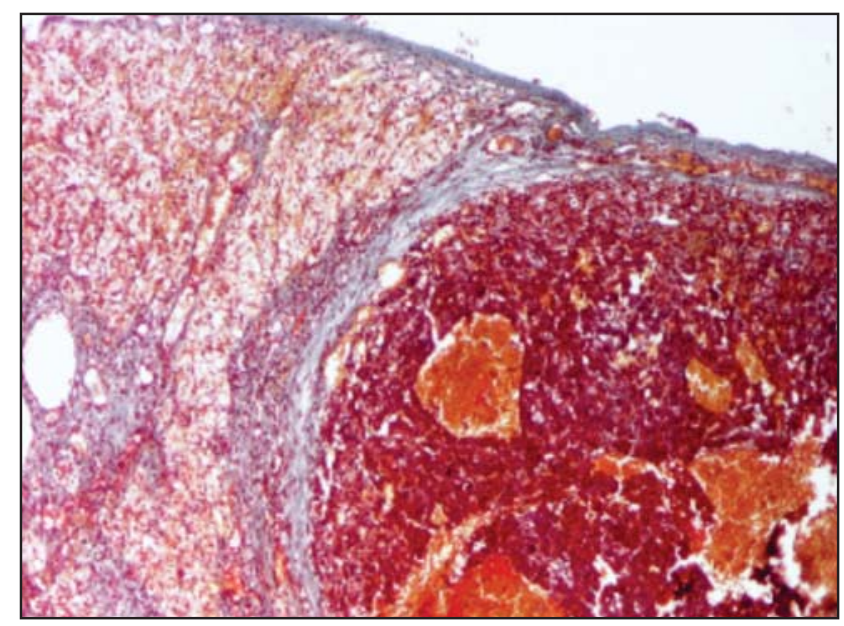

a

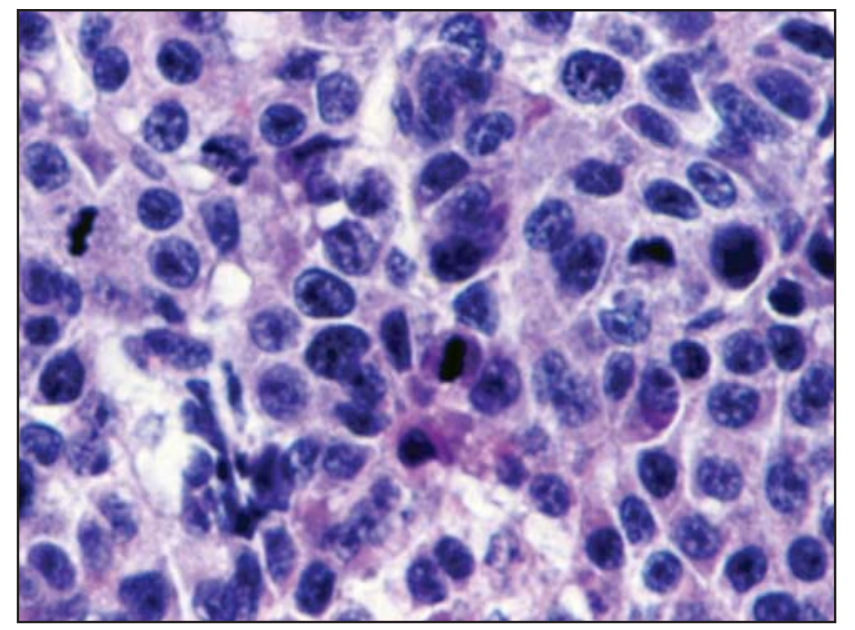

C

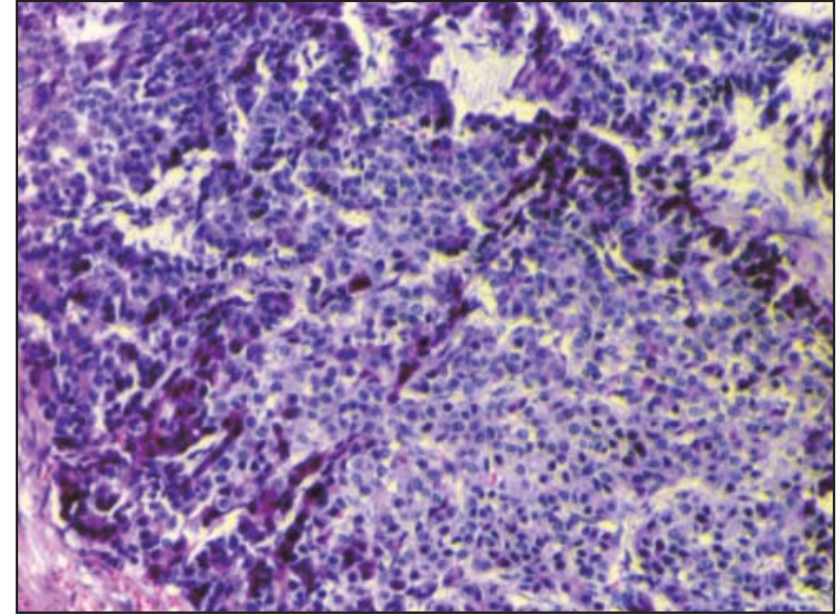

b

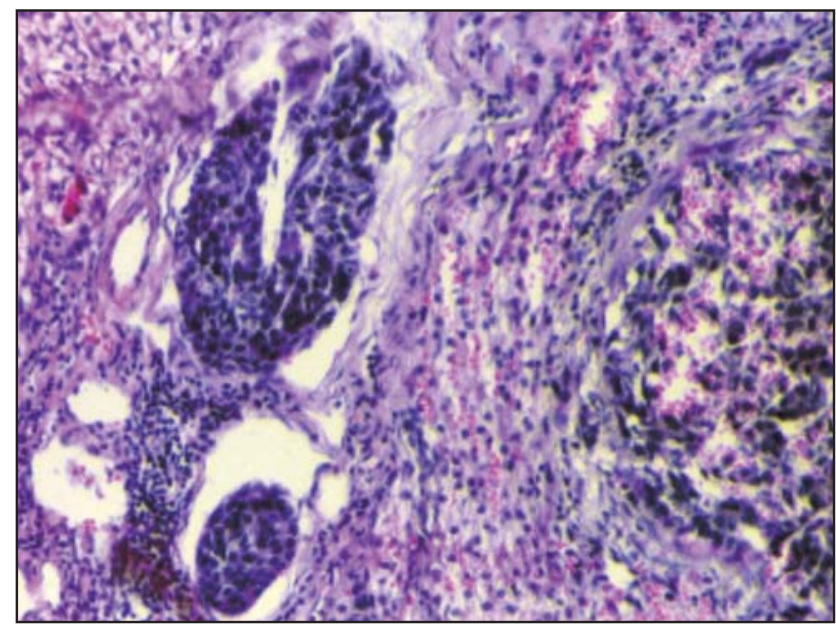

d

FIGURE 2 - a. Tumoral nodules; b. Vascular invasion; c. Mitoses, HE x 400; d. Embryonic and fetal hepatoblastoma, HE x 10

The anatomopathological examination confirmed the mixed epithelial hepatoblastoma with embryonic and fetal hepatocitary elements (Fig. 2 - a, b, c, d)

Based on the performed investigations, the final high-risk epithelial hepatoblastoma (with pulmonary metastases) diagnosis is established.

Later, the parents changed their mind and agreed to the chemotherapy according to the modified SIOPEL-4 protocol. After A1, A2 and A3 chemotherapy blocks have been completed, a thoraco-abdominal CT scan was performed on the patient, which confirmed a partial response of the pulmonary metastases to treatment and supported the continuation with the $\mathrm{C}$ chemotherapy block.

After the ninth chemotherapy session, in July 2014, shortly after being released from hospital, the child went through a febrile neutropenia episode, which evolved favorably under treatment with antibiotics, antimycotics, granulocyte growth factor, and blood transfusions. In September 2015, after the eleventh chemotherapy session, when the mod- ified SIOPEL-4 protocol was completed, the patient was re-examined, and the thoracic-abdominalpelvic CT scan outlined the absence of any signs of local recurrence and that of the secondary lesions, therefore a complete disease response to treatment.

\section{DISCUSSIONS}

Hepatoblastoma is a very rare tumor $(1-2 \%$ of the neoplasms in children), but it stands for $80 \%$ of all the liver malignant tumors in children $(3,4)$. The disease appears more frequently at Caucasian males under 3 years old. (5)

Hepatoblastoma etiopatogenesis is not completely elucidated. The tumor has been associated with some genetic hereditary syndromes in $15 \%$ of the cases. (6-9) Multiple environmental factors have been incriminated as hepatoblastoma causal factors, but so far it has only been proven that parents smoking status and low birth weight increase the risk of neoplasm appearance. (10-17) 
The most common symptoms and signs are: symptomatic or asymptomatic palpable abdominal mass (68\% of the cases), anorexia (23\%), abdominal pain (19\%), vomiting (11\%), or precocious puberty. The most frequent metastasis site is the lung, but there are also literary references to secondary lesions at the level of the heart, the central nervous system or the pulmonary artery. $(2,18-21)$

Biological screening can reveal high AFP values (usually $>50.000 \mathrm{ng} / \mathrm{ml}$, whereas values $>100.000$ suggest an advanced stage of the disease or metastases). $(22,23)$ In our case there was a decrease of the AFP level - from initially recorded and early post-operative values $>30.000 \mathrm{U} / \mathrm{L}$ - towards the normalization of the values in July.

Diagnosis methods are based primarily on imagistic investigation. Initially, an abdominal ultrasound is performed. CT and MRI have an essential role in neoplasm staging.

Differential diagnosis includes all types of liver masses: benign (hemangioma, hemangioendothelioma, focal nodular hyperplasia, liver post-traumatic cysts, congenital or echinococcal) or malignant (undifferentiated sarcoma, rhabdomyosarcoma, malignant mesenchymal tumor, hepatocellular carcinoma). (24)

Hepatoblastoma treatment is standardised. The SIOPEL group prefers chemotherapy before surgery and a few post-operative cycles, while the American Intergroup uses surgery per primam, and then post-operative chemotherapy. Chemotherapy is based on a protocol with high-dose cispatin in both groups. Hepatoblastoma with metastases is clasified as a high-risk disease. Therapeutic results for these tumors are considerably inferior (50\% survival rate for 3-year-olds) to standard-risk hepatoblastomas (limited to the liver or the 3 liver sections) which have a survival rate of $90 \%$ in 3 -yearolds. Therefore, the treatment of the high-risk hepatoblastoma is a challenge for the pediatric oncologist. The chemotherapy SIOPEL-4 protocol is used in this stage of the neoplasms. It introduces treatment with cisplatin (C) and doxorubicin (D) for the A1, A2, A2 blocks, and carboplatin (CA) and doxorubicin (D) fot blocks B and C.

In the presented case, the initiation of post-operative chemotherapy was decided according to the modified SIOPEL-4 protocol. Thus, all chemotherapy blocks which were supposed to be given before surgery were given post-operatively in the same doses. (Fig. 4)

With this treatment protocol, our patient had a complete tumor response. As far as we know, it is the first case with this particularity described in literature.

Liver transplant is a worth considering option for patients with an unresectable tumor and/or pulmonary metastases. The use of the liver transplant from the very beginning is preferable as the results are far more superior in terms of survival chances. However, for the liver transplant to be performed, the complete resection of all pulmonary metastases or their complete response to chemotherapy is necessary. Their absence must be confirmed by CT or

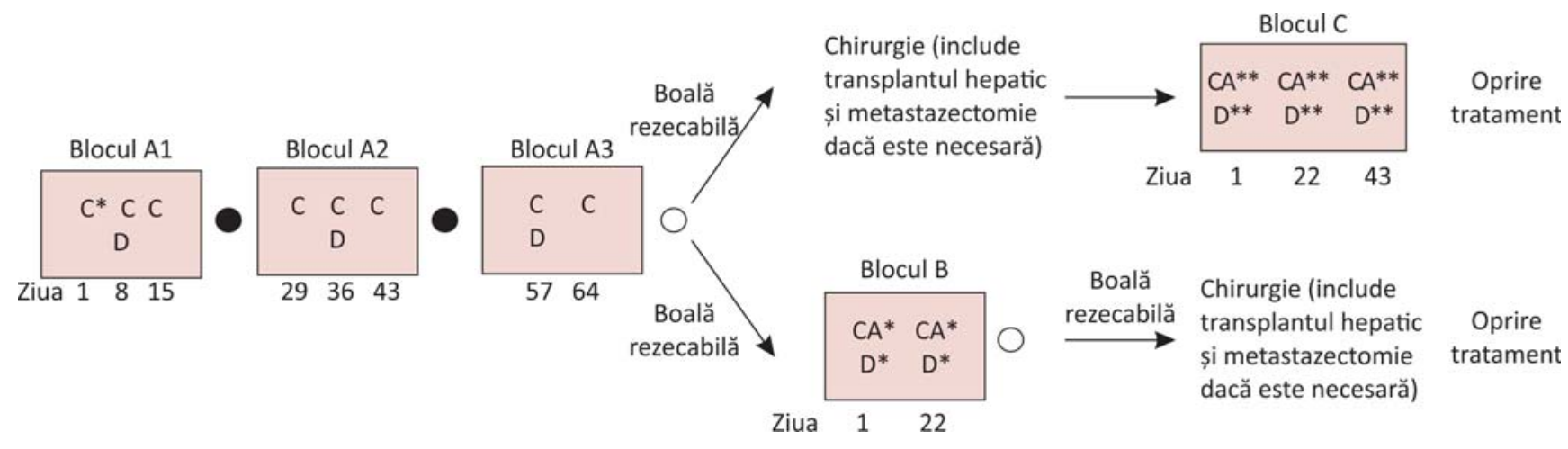

FIGURE 3. SIOPEL-4 protocol scheme, used in high-risk hepatoblastoma treatment

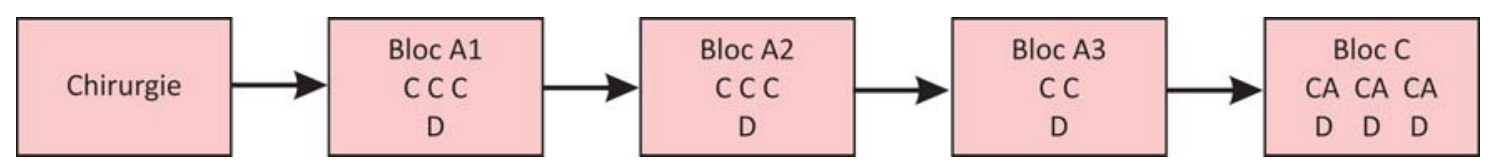

FIGURE 4. Modified SIOPEL-4 protocol used for our patient 
MRI investigations. Partial hepatectomy attempts with a high risk of incomplete resection should be avoided. (25)

\section{CONCLUSIONS}

Hepatoblastoma with pulmonary metastases has an unfavorable prognosis. At the moment, efforts of finding new therapeutic options meant to im- prove survival and quality of life are being made. In the shown case, a complete tumor response in a child with hepatoblastoma with pulmonary metastases was obtained. Therefore, modifying the chemotherapy protocols could represent one of the solutions for the prognosis improvement in these patients.

\section{REFERENCES}

1. Fuchs J., Rydzynski J., Hecker J. The influence of preoperative chemotherapy and surgical technique in the treatment of hepatoblastoma - a report from the German Cooperative Liver Tumours Studies HB 89 and HB 94. Eur J Pediatr. Surgery 2002; 12:255-261.

2. Meyers R.L., Tiao G., de Ville de Goyet J., Superina R., Aronson D.C. Hepatoblastoma state of the art: pre-treatment extent of disease, surgical resection guidelines and the role of liver transplantation. Curr OpinPediatr 2014 Feb; 26(1):29-36. doi:10.1097/ MOP. 0000000000000042.

3. Stringer M.D., Hennayake S., Howard E.R. et al. Improved outcome for children with hepatoblastoma. Br. J. Surg 1995; 82:386-391.

4. Stringer M.D. Liver tumours. Semin Pediatr Surg 2000; 9:196-208.

5. Willert J.R., Dahl G. Hepatoblastoma article.http://emedicine. medscape.com/ 986802-overview.

6. Tomlinson G.E., Kappler R. Genetics and epigenetics of hepatoblastoma. Pediatr. Blood Cancer 2012; 59:785-792.

7. DeBaun M.R., Tucker M.A. Risk of cancer during the first four years of life in children from the Beckwith-Wiedemann Syndrome Registry. J Pediatr 1998; 132:398-400.

8. Giardiello F.M., Offerhaus J.A., Krush A.J. et al. Risk of hepatoblastoma in familial adenomatous polyposis. J Pediatr 1991; 119:766-768.

9. Maruyama K., Ikeda H., Koizumi T. Hepatoblastoma associated with trisomy 18 syndrome: a case report and a review of the literature. Pediatr. Int 2001; 43:302-305.

10. Spector L.G., Johnson K.J., Soler J.T. et al. Perinatal risk factors for hepatoblastoma. Br J Cancer 2008; 98:1570-1573.

11. McLaughlin C.C., Baptiste M.S., Schymura M.J. et al. Maternal and infant birth characteristics and hepatoblastoma. Am J Epidemiol. 2006; 163:818-828.

12. Reynolds P., Urayama K.Y., Von Behren J. et al. Birth characteristics and hepatoblastoma risk in young children. Cancer 2004; 100:1070-1076.

13. Spector L.G., Puumala S.E., Carozza S.E. et al. Cancer risk among children with very low birth weights. Pediatrics 2009; 124:96-104.

14. Ansell P., Mitchell C.D., Roman E. et al. Relationships between perinatal and maternal characteristics and hepatoblastoma: a report from the UKCCS. Eur.J.Cancer 2005; 41:741-748.

15. Tanimura M., Matsui I., Abe J. et al. Increased risk of hepatoblastoma among immature children with a lower bith weight. Cancer 1998; 58:3032-3035.

16. Ikeda H., Matsuyama S., Tanimura M. Association between hepatoblastoma and very low birth weight: a trend or a chance? J. Pediatr 1997; 130:557-560.

17. Pu C.L., Guo C.B., Jin X.Q. et al. Retrospective analysis of maternal and infant birth features of hepatoblastoma patients. ZhonghuaGanZang Bing ZaZhi 2009; 17:459-461.

18. Chen W.J., Lee J.C., Hung W.T. Primary malignant tumour of liver in infants and children in Taiwan. J Pediatr Surg 1988; 23:457-461.

19. Kuo C.Y., Liu H.C., Chang M.H. et al. Hepatoblastoma in infancy and childhood: A clinical and pathological study of 32 cases. Acta Paediatr Sin 1991; 32: 79-87.

20. Brown B.F., Drehner D.M., Saldivar V.A. Hepatoblastoma: A rare pediatric neoplasm. Mil Med 1993; 158: 51-55.

21. Miron I., Aprodu G., Dumitra S. et al. Pseudopubertatea precoce Manifestare rară în hepatoblastom. Rev Rom Ped 2011; 60:76-82.

22. Hwang S.J., Luo J.C., Li C.P. et al.Thrombocytosis :a paraneoplastic syndrome in patients with hepatocellular carcinoma. World $\mathrm{J}$. Gastroenterol 2004; 10:2472-2477.

23. Perilongo G., Shafford E.A. Paediatric update. Liver tumours. Eur J Cancer 1999; 35(6): 953-959.

24. Perilongo G., Shafford E., Maibach R. et al. Risk adapted treatment for childhood hepatoblastoma; final report of the SIOPEL 2 study. Eur J Cancer 2004; 40:411-421.

25. Zsiros J., Brugieres L., Brock P. et al. Dose-dense cisplatin-base chemotherapy and surgery for children with high-risk hepatoblastoma (SIOPEL-4): a prospective, single-arm, feasibility study. Lancet Oncol 2013. http://dx.doi.org/10.1016/S1470-2045(13)70272-9. 